\title{
Considerations for Combining High-Protease Extracts in Immunotherapy Vaccines
}

\author{
Carol M. Lilley ${ }^{1}$, Donna J. Rekkerth ${ }^{2 *}$, Brian F. Teske ${ }^{3}$ \\ ${ }^{1}$ American Society of Allergy Nurses, Albany, USA \\ ${ }^{2}$ GREER $^{\circledR}$ Laboratories, Inc., Lenoir, USA \\ ${ }^{3}$ FORCE Communications, Indianapolis, USA \\ Email: ${ }^{\text {drekkerth@greerlabs.com, asan@peak.org, bteske@forcemed.com }}$
}

Received 10 January 2014; revised 11 March 2014; accepted 31 March 2014

Copyright (C) 2014 by authors and Scientific Research Publishing Inc.

This work is licensed under the Creative Commons Attribution International License (CC BY). http://creativecommons.org/licenses/by/4.0/

(c) (i) Open Access

\begin{abstract}
Current practice parameters state that extracts rich in proteases, such as fungal and insect extracts, can be combined during preparation of allergy immunotherapy vaccines. However, until recently, this guideline has not been the subject of investigation. Scientists now have data that shed light on high-protease allergenic extract mixtures used in allergy immunotherapy. A study published in Annals of Allergy, Asthma \& Immunology in 2012 reports on the compatibility of combining fungal and insect extracts and emphasizes the importance of understanding how protease activities and total glycerin levels in allergy extracts can affect the stability of allergy immunotherapy vaccine mixtures. This research provides a critical assessment of the mixing compatibilities of several well-characterized high-protease extracts and may influence future immunotherapy practice parameters and immunotherapy extract preparation guidelines.
\end{abstract}

\section{Keywords}

Immunotherapy; Allergen; Extract; Compatibility; Protease

\section{Introduction}

Allergy immunotherapy has been in practice for over a century. In 1911, Leonard Noon and John Freeman were the first to use subcutaneous immunotherapy (SCIT) in humans for the treatment of "hay fever" [1]. Since then, SCIT has been the subject of numerous controlled studies and has proven over time to be efficacious for the treatment of a variety of allergic disorders, including allergic rhinoconjunctivitis, allergic asthma, and hypersensitivity to stinging insects [2]. SCIT results in a modification of the immune system that can provide long-term

*Corresponding author. 
benefits for patients who wish to control the underlying causes of their allergy symptoms [3]. SCIT involves systematic administration of allergenic extracts at effective doses. Controlled, gradual introduction of a known allergen allows the immune system to build up tolerance over time-a process that ultimately desensitizes the patient to the allergen [3]. This disease-modifying approach is often implemented when patients are no longer able to control their allergies with pharmacotherapy or avoidance measures, but it can also be discussed with patients early on in treatment, particularly if patients are affected by the adverse events that accompany chronic therapy with antihistamines or prefer to reduce the use of these medications.

\section{Degradation of Allergens in Mixtures}

Some patients are diagnosed with immunoglobulin E (IgE)-mediated hypersensitivities to a variety of allergens and require treatment with multiple allergenic extracts. In most of these cases, healthcare providers have to decide whether or not to combine the allergenic extracts into 1 treatment vial to reduce the number of injections given or to prepare 2 or more treatment vials, which would require multiple injections. Knowledge of allergen compatibilities and total glycerin content in these extract mixtures can influence whether the allergens can be formulated together or whether they will need to be separated. Because of this, understanding allergen compatibility and the impact of glycerin content on stability of extract potencies in these mixtures can facilitate preparation of consistent allergen mixtures and delivery of effective and safe allergen doses in SCIT vaccines.

Allergen stability and potency can be affected by storage conditions, handling, fluctuations in temperature, changes in $\mathrm{pH}$, variations in glycerin content, and compatibility of allergenic extracts [4] [5]. Enzymes called proteases naturally occur in certain allergenic extracts and are a major reason for extract incompatibilities [4]. Proteases cause degradation of proteins through cleavage of peptide bonds and can ultimately compromise the stability of the antibody-binding activities of proteins in extracts used for immunotherapy. Some allergenic extracts (e.g., pollen, mite, animal) are classified as low-protease or protease-susceptible extracts, whereas most fungal and insect extracts are classified as high-protease extracts [6]. To date, allergen mixology research has focused primarily on the effects of combining low-protease with other low-protease allergenic extracts or lowprotease with high-protease allergenic extracts to determine the effects of mixing on shelf life and stability of allergens [6].

\section{Insights into High-Protease Extracts}

In a study [6] published in the Annals of Allergy, Asthma and Immunology, the official publication of the American College of Allergy, Asthma \& Immunology, researchers asked the question: What if 2 highly proteolytic allergenic extracts were combined in the same extract mixture? The researchers thought it was particularly important to answer this question because, while immunotherapy practice parameters state that these extracts can be combined, data to support this practice had not been published prior to this paper. Two high-protease extracts prepared from the fungus Alternaria alternata and the German cockroach (Blatella germanica) were used in this study. The allergenic extracts were combined with other protease-rich fungal and insect extracts at product concentrations corresponding to dose levels recommended for maintenance immunotherapy injections. The stability of the allergens was tested in fungal-fungal, fungal-insect, and insect-insect mixture combinations. The allergenic extract mixtures were stored in $10 \%, 25 \%$, and $50 \%$ glycerin solutions at $2^{\circ} \mathrm{C}-8^{\circ} \mathrm{C}$, and data were collected over a period of 2 - 12 months.

After mixing and storage, the stability of the major Alternaria allergen Alt a 1 and major cockroach allergens Bla g 1 and Bla g 2 were measured using a panel of in vitro testing methods. Other minor allergens were also measured for stability and compatibility in these mixtures. Because each assay measures a slightly different property of the allergens, a panel of three different analytical methods was used to fully characterize the allergen stability. Human IgE ELISA inhibition assays (ELISA: enzyme-linked immunosorbant assays), quantitative tests which measure the ability of patients' IgE to recognize and bind to allergen, and thus whether the natural structure of the antibody binding sites are maintained, were performed as previously described [7] [8]. Validated, double binding ELISAs were used to quantitatively measure the levels of the major allergens Alt a 1, Bla g 1, and Bla g 2 in the extracts (detailed experimental methods previously described [6]). Finally, to identify all the proteins in the complex extract mixtures that are recognized by human IgE, qualitative immunoblots were performed as described previously [6]-[9]. Because these assays measure different molecular structures or conformations, use of multiple analytical procedures can provide more diverse and objective information on the phys- 
ical and biochemical integrity of allergens than individual methods alone [6]. Importantly, the recovery of both the major and minor allergenic components was compared to a control sample at the identical concentrations of target allergens and glycerin.

The most significant susceptibilities were found when the high-protease fungal and insect extracts were combined across phylogenetic groups (e.g., fungi with insects) however some mixtures were found to be more risky than others. For example, when Alternaria extracts were combined with American cockroach extracts, the recovery of the major Alt a 1 allergen was significantly diminished at all storage conditions tested. Compared to the recovery of Alt a 1 in Alternaria-American cockroach extract mixtures, when Alternaria extracts were combined with German cockroach or fire ant extracts, the Alt a 1 allergen was recovered at a higher rate. The relative potency of the other minor allergens in the Alternaria extract was measured by IgE binding assay. The recovery of minor allergens was significantly reduced when combined with fire ant extract, an effect that was improved by increasing the glycerin concentration. It was also noted that the recovery of the Alternaria minor allergens was unchanged when Alternaria extracts were combined with American or German cockroach extracts. A similar line of experimentation was carried out for German cockroach extracts; when mixed with fungal extracts, the major allergens Bla g 1 and Bla g 2 were recovered at a high rate in all extract mixtures tested. However, the minor allergen recovery was significantly reduced when combined with fungal species. The most drastic reduction in minor allergen recovery was found when German cockroach extracts were combined with Penicillium and Aspergillus extracts. This reduction was also seen when German cockroach extracts were combined with Alternaria and Bipolaris species, albeit to a lesser extent.

Overall, researchers found that combining high-protease-containing extracts within the same phylogenetic group (e.g., fungal-fungal or insect-insect) did not significantly change the quality of the major or minor allergens in the mixture [6]. However, when allergenic extracts were combined across species, stability of the allergens was compromised, and some mixture combinations were found to be more risky than others.

\section{Putting It into Practice}

From a clinical perspective, it is important for healthcare providers to understand that the stability of allergens in some fungal-insect extract mixtures can be compromised due to incompatibilities. Current immunotherapy practice parameters may need to be updated to accommodate the recommendations of more recently published findings. Therefore, it may be necessary to prepare more than 1 treatment vial to create more stable mixtures for SCIT. If more than 1 treatment vial is prepared, the patient will need more than 1 allergy injection. If more than 1 treatment vial is not desired, it may be possible to alleviate some of these compatibility problems by increasing the total glycerin content in the vial. The decision of whether or not to combine allergen extracts that are not compatible will depend on clinical judgment and an integration of clinical knowledge, external clinical evidence from published guidelines, and individual patient needs and wishes in regards to the number of allergy injections they will receive. Common questions healthcare providers often ask are:

- What if more than 1 shot will cause a patient to refuse immunotherapy, and 1 or 2 of the allergens required are incompatible?

- Isn't there a way in which allergens can be combined together in special situations even if they are not compatible?

- If research has shown that non-compatible allergens are more stable in higher glycerin levels, could more glycerin be added to the treatment vial to increase stability when combining allergens that are not compatible?

These are difficult questions to answer because, ideally, allergens that are not compatible should be separated into different treatment vials, as was recommended in the 2012 research. However, research has demonstrated that increasing the total glycerin content in a vial may increase stability of the vial even if the allergenic extracts are non-compatible [6]. Therefore, if a patient is only allergic to a few allergens and is only willing to receive 1 allergy injection, increasing the total glycerin in the treatment vial to at least $25 \%$ may increase stability and compatibility of the mixture [6]. If glycerin content is greater than $25 \%$ in the treatment vial, the healthcare provider may need to weigh the risk of increasing local irritation at the injection site. Ideally, high- and low-protease allergenic extracts should be separated into more than 1 treatment vial whenever possible, but in certain circumstances, the total glycerin content can be increased to $25 \%$ to increase the stability of the treatment vial in order to reduce the number of injections required. 


\section{Conclusion}

SCIT is an important disease-modifying treatment option for allergy patients. Maintaining stability of the specific allergens in the patient treatment vial is critical to achieving therapeutic allergen preparations. Contrary to practice parameters, allergen compatibilities can be compromised in some fungal-insect extract mixtures, which may decrease the overall therapeutic effect for the patient. Clinical judgment needs to be used when deciding which allergenic extracts will be combined, the total glycerin content, and how many treatment vials will be prepared to meet the needs of each individual patient.

\section{Conflict of Interest}

D.J.R., serves as a medical affairs specialist at GREER® Laboratories, Inc. B.F.T. serves as a scientific innovation manager at FORCE Communications and was funded by GREER ${ }^{\circledR}$ Laboratories. No conflict of interest is declared for C.M.L.

\section{References}

[1] Ring, J. and Gutermuth, J. (2011) 100 Years of Hyposensitization: History of Allergen-Specific Immunotherapy (ASIT). Allergy, 66, 713-724. http://dx.doi.org/10.1111/j.1398-9995.2010.02541.x

[2] Cox, L., Calderon, M. and Pfaar, O. (2012) Subcutaneous Allergen Immunotherapy for Allergic Disease: Examining Efficacy, Safety and Cost-Effectiveness of Current and Novel Formulations. Immunotherapy, 4, 601-616. http://dx.doi.org/10.2217/imt.12.36

[3] Burks, A.W., Calderon, M.A., Casale, T., et al. (2013) Update on Allergy Immunotherapy: American Academy of Allergy, Asthma \& Immunology/European Academy of Allergy and Clinical Immunology/PRACTALL Consensus Report. Journal of Allergy and Clinical Immunology, 131, 1288-1296. http://dx.doi.org/10.1016/j.jaci.2013.01.049

[4] Esch, R.E. and Grier, T.J. (2011) Allergen Compatibilities in Extract Mixtures. Immunology and Allergy Clinics of North America, 31, 227-239. http://dx.doi.org/10.1016/j.iac.2011.02.009

[5] Nelson, H.S. (1981) Effect of Preservatives and Conditions of Storage on the Potency of Allergy Extracts. Journal of Allergy and Clinical Immunology, 67, 64-69. http://dx.doi.org/10.1016/0091-6749(81)90047-6

[6] Grier, T.J., LeFevre, D.M., Duncan, E.A., Esch, R.E. and Coyne, T.C. (2012) Allergen Stabilities and Compatibilities in Mixtures of High-Protease Fungal and Insect Extracts. Annals of Allergy, Asthma \& Immunology, 108, 439-447. http://dx.doi.org/10.1016/j.anai.2012.04.012

[7] Grier, T.J., LeFevre, D.M., Duncan, E.A. and Esch, R.E. (2007) Stability of Standardized Grass, Dust Mite, Cat, and Short Ragweed Allergens after Mixing with Mold or Cockroach Extracts. Annals of Allergy, Asthma \& Immunology, 99, 151-160. http://dx.doi.org/10.1016/S1081-1206(10)60639-4

[8] Grier, T.J., LeFevre, D.M., Duncan, E.A. and Esch, R.E. (2009) Stability and Mixing Compatibility of Dog Epithelia and Dog Dander Allergens. Annals of Allergy, Asthma \& Immunology, 103, 411-417. http://dx.doi.org/10.1016/S1081-1206(10)60361-4

[9] Kurien, B.T. and Scofield, R.H. (1997) Multiple Immunoblots after Non-Electrophoretic Bidirectional Transfer of a Single SDS-PAGE Gel with Multiple Antigens. Journal of Immunological Methods, 205, 91-94. http://dx.doi.org/10.1016/S0022-1759(97)00052-5 